\section{Índice de vulnerabilidad de los hogares en el municipio de Pasto, Colombia, 2012}

\author{
Family vulnerability index in the municipality of \\ Pasto, Colombia, 2012
}

Índice de vulnerabilidade das famílias no
Município de Pasto, Colômbia, 2012

\section{Resumen}

El objetivo del estudio fue medir la tasa de vulnerabilidad de las familias en el municipio de Pasto, Colombia. En una muestra de 270 familias de todos los estratos socioeconómicos, 239 en áreas urbanas y 31 en las zonas rurales, se realizó una investigación de carácter confidencial, utilizando el índice de vulnerabilidad, consistente en cinco dimensiones: demográfica, social, económica, ambiental y geográfica, así como previsión y prevención. Las familias estrato 1 y 2, así como los hogares del centro, oeste, noroeste y nordeste del área urbana del municipio, presentaron alta vulnerabilidad, incluso más que aquellas en áreas rurales. Un 50\% de las familias estaban localizadas dentro de baja vulnerabilidad y un $42 \%$ estaban en nivel medio. Las familias más pobres fueron las más vulnerables, sin embargo, las familias pobres también eran vulnerables en el municipio de Pasto.

Pobreza; Poblaciones Vulnerables; Condiciones Sociales
Arsenio Hidalgo-Troya 1

Gissela Fernanda Guerrero-Díaz 2

Vivian Liseth Estupiñan-Ferrín 3

Anderson Rocha-Buelvas 3

doi: 10.1590/0102-311X00122315

\author{
Correspondencia \\ A. Rocha-Buelvas \\ Maestría en Salud Pública y Desarrollo Social, Fundación \\ Universitaria del Área Andina. \\ Carrera 14A, \#71-19, Bogotá D.C. 11001000, Colombia. \\ rochabuelvas@gmail.com \\ 1 Facultad de Ciencias Exactas y Naturales, Universidad de \\ Nariño, Pasto, Colombia. \\ 2 Facultad de Ciencias Económicas, Universidad de Nariño, \\ Pasto, Colombia. \\ 3 Maestría en Salud Pública y Desarrollo Social, Fundación \\ Universitaria del Área Andina, Bogotá, Colombia.
}




\section{Introducción}

La pobreza no necesariamente es sinónimo de vulnerabilidad, ya que existe la probabilidad de que personas catalogadas como no pobres puedan verse afectadas por los cambios de una sociedad con crecientes señales de inseguridad, incertidumbre y desprotección ${ }^{1}$. Se ha definido la vulnerabilidad como el proceso multidimensional que confluye en el riesgo o probabilidad de que un individuo, hogar o comunidad pueda resultar dañado, herido o lesionado, frente a cambios o permanencia de situaciones internas y/o externas 2. La vulnerabilidad se comprende en cuatro ámbitos: trabajo; capital humano; activos productivos y activos intangibles, tales como: las relaciones sociales y el capital social. De modo que la vulnerabilidad social, está íntimamente relacionada con el mercado de trabajo; la prestación de los servicios sociales en educación, salud y previsión social; las modificaciones de las formas tradicionales de organización y participación social y las debilidades de pequeñas empresas 2,3. No obstante, los estudios sobre vulnerabilidad social están tendiendo a realizarse en el contexto del cambio climático, dada su influencia en la producción de alimentos, en la cultura y la configuración demográfica de los territorios, según indicadores ecológicos 4,5,6,7,8,9,10.

La Comisión Económica para América Latina y el Caribe (CEPAL), en los años 1980, midió por primera vez la pobreza en Colombia, recomendándose desde entonces el índice de Necesidades Básicas Insatisfechas (NBI) para caracterizar la pobreza 11. La medición de vulnerabilidad tiene antecedentes en el Programa de las Naciones Unidas para el Desarrollo (PNUD), que en el año 2004 diseña el Índice de Riesgos de Desastre (IRD), con el fin de medir y comparar entre países, los niveles relativos de exposición física a la amenaza, la vulnerabilidad y los riesgos, definiendo la vulnerabilidad como la situación o proceso en el que intervienen factores físicos, sociales, económicos y ambientales, que determinan cuáles y cuántos daños podría acarrear una determinada amenaza natural 12. Contradictoriamente, la Universidad Autónoma de Madrid (España) en el año 2006 estimó el grado de vulnerabilidad a los ciudadanos que se encuentran sometidos, en materia de desprotección social, utilizando como referente a los países de la Organización para la Cooperación y el Desarrollo Económicos (OCDE) 13. Hallazgos sobre vulnerabilidad en Colombia reportan que, por un lado alrededor del 20\% de los hogares colombianos, además de encontrarse en una condición de pobreza, tienen una alta probabilidad de permanecer en ella y, por otro lado, que cerca de un $18 \%$ de los hogares vulnerables, paradójicamente, no eran pobres; de modo que las políticas públicas en Colombia están orientadas a combatir la pobreza y no la vulnerabilidad de los hogares ante la pobreza futura 14 .

El índice de vulnerabilidad es un instrumento útil para la focalización de los programas sociales y una guía para la formulación de las políticas públicas, tanto a nivel municipal, como a nivel departamental y nacional. Al respecto, el Departamento Nacional de Planeación de Colombia (DNP) 15 (p. 7), en uno de sus estudios, define a la focalización como "la discriminación positiva en favor de un grupo de personas que cumplen unas características previamente establecidas", ya que la focalización se efectúa definiendo instrumentos, a partir de los cuales se identifican los beneficiarios del gasto social. Así, pueden distinguirse varios métodos de focalización, entre los más generales se encuentran: la evaluación individual, la focalización geográfica y la auto-focalización. El índice de vulnerabilidad propuesto, dadas sus características, se enmarca dentro del método de focalización individual que se realiza a través del método de los proxy means test, que a su vez parten de variables consideradas relevantes y verificables 16 .

El municipio de Pasto es la capital del Departamento de Nariño, en el suroccidente colombiano, una ciudad ubicada en la cordillera occidental y cercana a la frontera con Ecuador. Su área urbana está dividida en 12 comunas y la zona rural está compuesta por 17 corregimientos. La economía está basada en la producción de alimentos, artesanía, micro-industrias y comercio inter-fronterizo; también cuenta con más de un $90 \%$ de alfabetismo y cobertura de servicios públicos, mientras el nivel de ingreso per cápita promedio está por debajo del 50\% 17. Las características de esta población hicieron propicio plantearse la medición del índice de vulnerabilidad de hogares urbanos y rurales del municipio de Pasto en el año 2012. 


\section{Materiales y métodos}

Estudio descriptivo de corte transversal que se construyó, validó y aplicó, a través de una encuesta índice (Figura 1). La población y muestra fue obtenida de las proyecciones demográficas de la población del municipio de Pasto para el año 2012 (423.217 habitantes, de los cuales el 88,5\% pertenece a la zona urbana y el 11,5\% restante a la zona rural), según el Departamento Administrativo Nacional de Estadísticas (DANE), asimismo, se tuvo en cuenta el promedio de integrantes por hogar en el municipio de Pasto, según el Boletín del Censo General de 2005 18, de 3,9. Se calculó la población, dividiendo el número promedio de personas por hogar en el municipio de Pasto, siendo el número de hogares para el año 2012 de 108.517 hogares. La muestra cuenta con la siguiente formula estadística:

$$
\mathrm{n}=\frac{\mathrm{N} * \mathrm{Z}^{2 *}\left(\mathrm{p}^{*} \mathrm{q}\right)}{\mathrm{e}^{2 *}(\mathrm{~N}-1)+\mathrm{Z}^{2 *}\left(\mathrm{p}^{*} \mathrm{q}\right)}
$$

Así:

$$
\begin{aligned}
& n=\frac{108.517 *(1,645)^{2 *}(0,5)^{*}(0,5)}{\left[(0,05)^{2 *} 108.516\right]+(1,645)^{2 *}(0,5)^{*}(0,5)} \\
& n=\frac{73.412,43}{271.966} \\
& n=269,93 \approx 270
\end{aligned}
$$

Donde, $\mathrm{n}=$ tamaño de la muestra a calcular; $\mathrm{N}$ = es la población total (108.517 hogares del municipio de Pasto para el año 2012); $Z=90 \%$ de confianza $\longrightarrow$ 1,645; es el valor crítico encontrado en la tabla de la distribución normal; $\mathrm{p}=$ probabilidad de éxito: 0,$5 ; \mathrm{q}=$ probabilidad de fracaso: 0,$5 ; \mathrm{E}=$ error máximo: 0,05.

El resultado de la muestra para la aplicación de la encuesta fue de 270 hogares del municipio de Pasto, de los cuales 239 se aplicaron en el sector urbano, a partir de un proceso probabilístico proporcional y estratificado, y 31 encuestas en el sector rural, a través de un proceso probabilístico por conglomerados. En el caso de los hogares urbanos, los de estrato 1 correspondieron a 43 encuestas, el 2 a 93, el 3 a 78, el 4 a 20, el 5 a 5 y para el 6 no hubo encuestas.

La encuesta constó de cinco dimensiones, una demográfica (D1) que se refiere a la estructura del hogar y las condiciones físicas del mismo para determinar las capacidades del hogar para hacer frente a las situaciones que pueden afectar el núcleo familiar y la dependencia demográfica al interior del mismo. En esta dimensión se dio especial importancia a la caracterización del jefe de hogar, pues este es el responsable y encargado de la consecución de recursos para el bienestar del hogar. La otra dimensión fue la social (D2) que se refirió a la educación de los miembros del hogar, acceso a sistemas de salud, condiciones habitacionales, acceso a los servicios públicos y las relaciones sociales que dan lugar al capital social. La tercera dimensión fue la económica (D3) que se refirió a la situación laboral de los miembros del hogar, los niveles de ingreso, la tenencia de activos (riesgo ex-ante). La dimensión ambiental y geográfica (D4) que pretendió establecer, si la ubicación urbana o rural de un hogar implica mayor o menor nivel de vulnerabilidad. Y, finalmente, la dimensión de previsión y percepción (D5) que se refirió a la existencia de condiciones y herramientas que tiene un hogar para afrontar las perturbaciones actuales y futuras, y su percepción sobre el apoyo del gobierno y el manejo de sus políticas públicas 3,11,13,19.

La encuesta constó de 65 variables explicativas de los diferentes niveles de vulnerabilidad de los hogares, para lo cual D1 contó con 11 variables; D2 con 32 variables; D3 con 9 variables; D4 con 3 variables y D5 con 10 variables. Tras la aplicación de procedimientos estadísticos, con fines de validación, se utilizaron 31 variables, después de la aplicación de pruebas estadísticas que validaron el uso de la encuesta (Figura 2). Las variables categóricas fueron codificadas, asignándoles valores numéricos de manera ordinal, donde los valores asignados al grado de vulnerabilidad oscilaron entre 1 a 5; siendo 1 el nivel más bajo de vulnerabilidad. Mientras que para las variables numéricas, se utilizaron algunas 
Figura 1

Propuesta metodológica para la construcción del índice de vulnerabilidad (Fase 1).

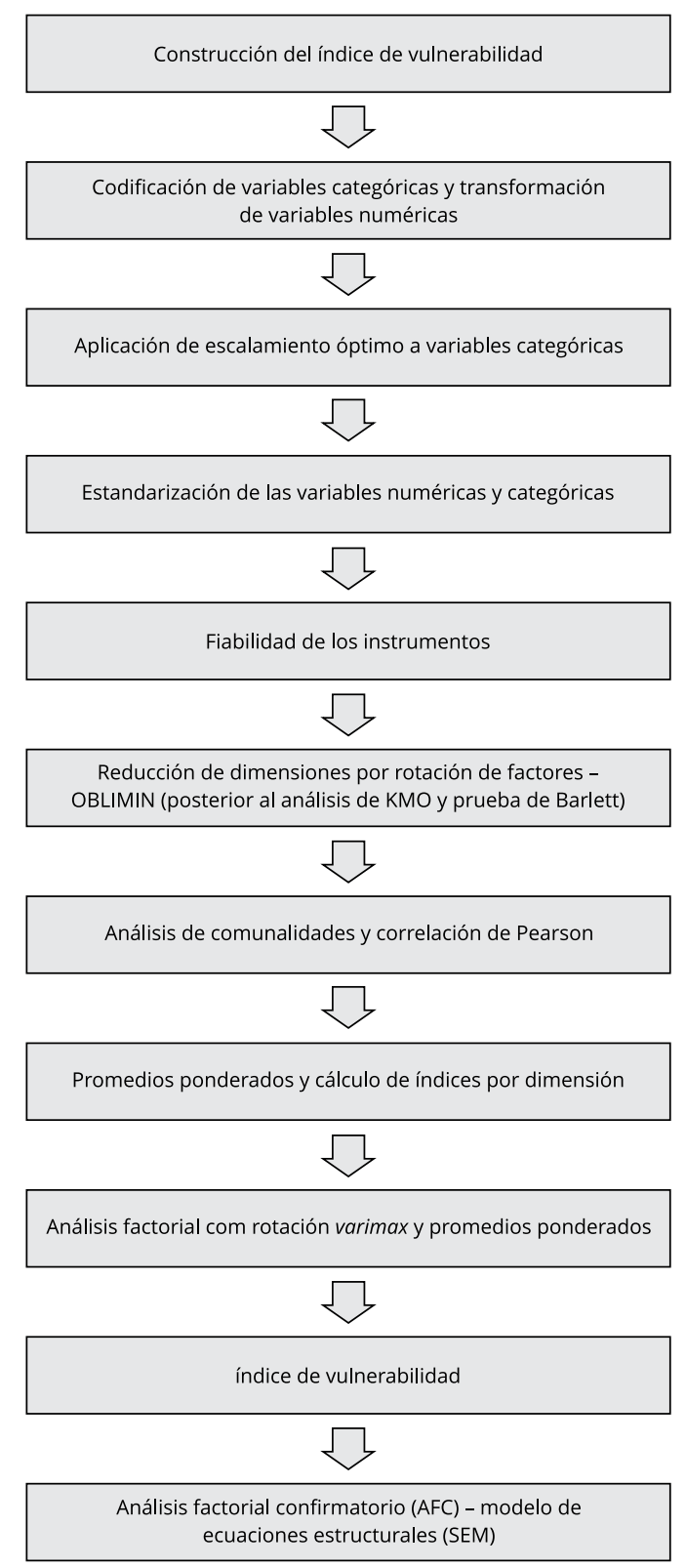

KMO: Kaiser Meyer Olkin; OBLIMIN: reducción de dimensiones por rotación de factores. 
fórmulas matemáticas para calcular el valor correspondiente para cada observación. En este sentido, las variables edad y situación ocupacional se transformaron en dependencia económica (DE) y dependencia demográfica (DD) respectivamente, a través de las siguientes relaciones:

$$
\begin{aligned}
& D E=\frac{\text { Inactivos }+ \text { Desempleados }+ \text { Menores de } 14 \text { años }}{\text { Empleados }+ \text { Independientes }} \\
& D D=\frac{\text { Miembros } \geq 60 \text { años }+ \text { Miembros } \leq 14 \text { años }}{\text { Miembros entre } 15 \text { y } 59 \text { años }}
\end{aligned}
$$

De esta manera, los resultados de la DE y DD tomaron valores entre 0 y 1 , donde 0 representa la ausencia de dependientes en el hogar, bien sea a nivel demográfico o económico. Se encuentran entre las variables transformadas con promedios correspondientes a preguntas con múltiples opciones de respuesta: activos del hogar, ingresos adicionales, seguros, problemas en el hogar, espacios públicos, servicios públicos básicos y complementarios, desastres naturales, problemas ambientales y nivel educativo del hogar. En esta última, el promedio se obtuvo teniendo en cuenta solamente los años de escolaridad de los miembros y a los mayores de 16 años. Por otra parte, la variable estabilidad laboral se creó a partir del uso de la información de dos variables distintas: situación ocupacional y tipo de trabajo. Así, se estableció una relación entre el número de personas que trabajan, y que se catalogan como trabajadores ocasionales, y la cantidad total de ocupados (empleados e independientes) en el hogar. El resultado de esta variable indica la proporción de trabajadores ocasionales en el hogar. De esta manera, valores cercanos a 1, indicaron que existe mayor inestabilidad laboral y, por tanto, mayor vulnerabilidad. También hubo un grupo cuya trasformación consistió en calcular proporciones para cada opción de respuesta, dividiendo la frecuencia de respuesta de una opción por el número total de miembros del hogar. Este es el caso de las variables: embarazo juvenil, desnutrición, enfermedad crónica, limitaciones físicas, nacimientos, fallecimientos, afiliados a pensión, destrezas (saber leer, saber escribir, saber usar un computador y saber navegar por Internet), salud, consecución de empleo, participación en grupos sociales y obtención de beneficios.

De modo que se construyó el índice de vulnerabilidad a partir de la (i) codificación de variables categóricas y transformación de variables numéricas, a la (ii) aplicación de escalamiento óptimo a variables categóricas; a la (iii) estandarización de las variables numéricas y categóricas; a la (iv) fiabilidad del instrumento por medio del cálculo del índice alfa de Cronbach para establecer la fiabilidad del instrumento, a través del promedio de las correlaciones entre los ítems, obteniendo un resultado aceptable de 0.76 , que valida el uso del instrumento para la recolección de datos; a la (v) reducción de dimensiones por rotación de factores (OBLIMIN), donde se compara la magnitud de los coeficientes de correlación observados, con la magnitud de los coeficientes de correlación parcial con la prueba de Kaiser Meyer Olkin (KMO), cuyo valor fue de 0,7 , indicando que hay una aceptable adecuación muestral y, junto con el test de esfericidad de Bartlett con $\mathrm{p}<0,05$, plantean la factibilidad del análisis factorial tipo $\mathrm{R}$, para determinar qué variables son más significativas en la explicación de las dimensiones que abarcan la vulnerabilidad. Este análisis factorial demostró que era necesario prescindir de algunas variables; por ejemplo, D1 poseía las mayores cargas factoriales en 7 de las 11 variables, razón por la cual se prescinde de las variables: embarazo prematuro, emigración, nacimientos y fallecimientos. Asimismo, se realizó un análisis de elementos en común para asegurar que las variables alcancen niveles aceptables de explicación, donde se determinó que las variables emigración y nacimiento de D1 con $\mathrm{p}<0,50$ eran carentes de explicación suficiente; mientras el análisis de correlación de Pearson determinó que las variables inmigración y desplazamiento de D1 se eliminaran, porque tienen una correlación mayor de 0,8. Luego con el cálculo de (vi) promedios ponderados y el cálculo de índices por dimensión, se consideraron como explicativas y relevantes para el análisis 31 variables de las 65 variables iniciales. Posteriormente, con el (vii) análisis factorial, con método de rotación ortogonal (varimax), se identificaron las mayores cargas factoriales absolutas para cada dimensión, en cada factor de la matriz de componentes rotados con varimax, luego, se sumaron los índices de las dimensiones multiplicadas por la varianza $(\lambda i)$ de su componente para obtener el índice de 
Figura 2

Diagrama de secuencias de relaciones causales por dimensión.

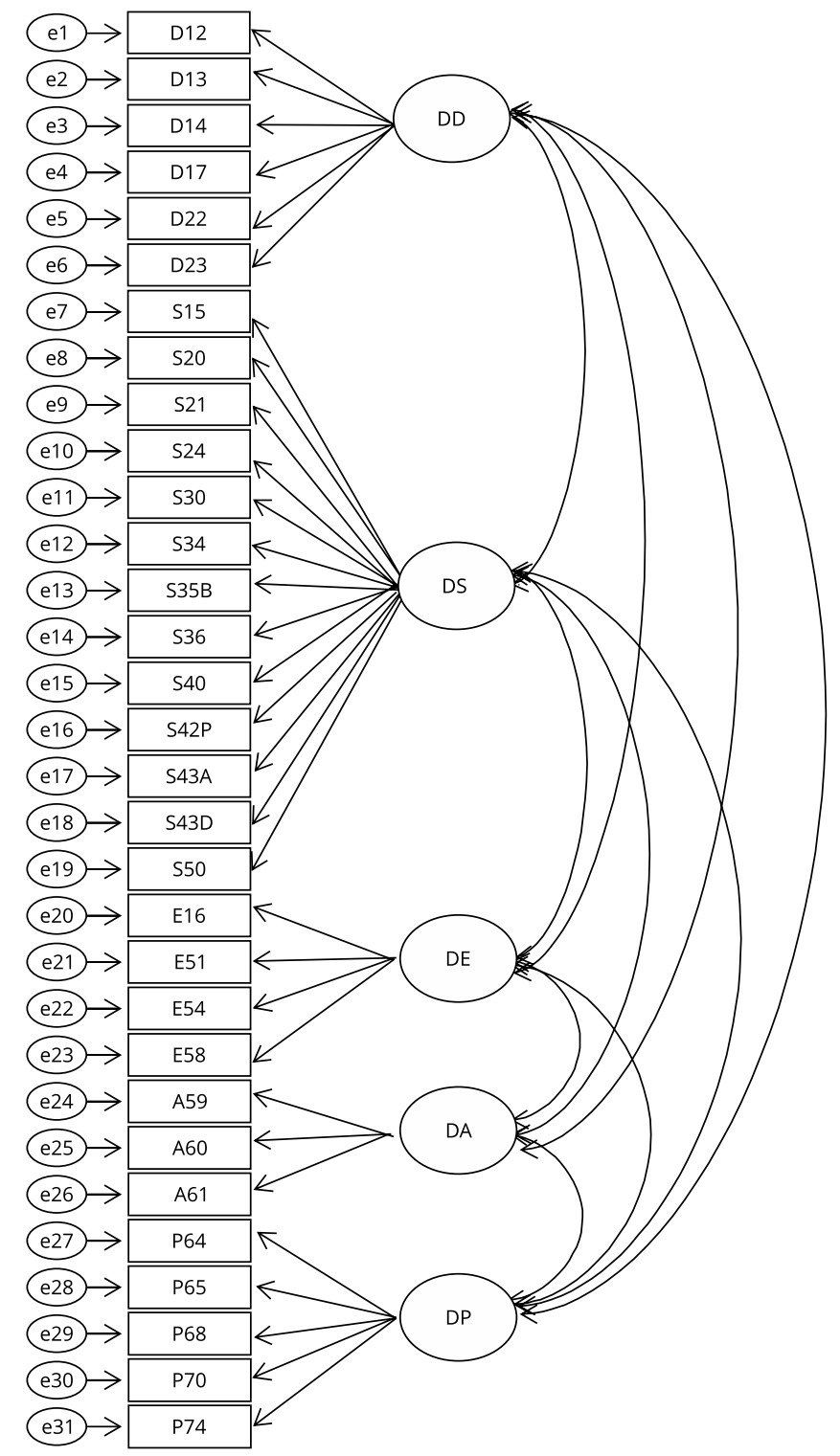

DA: dimensión ambiental-geográfica; DD: dimensión demográfica; DE: dimensión económica; DP: dimensión de previsión y percepción; DS: dimensión social. 
vulnerabilidad de los hogares. Finalmente, se obtuvieron los niveles de vulnerabilidad, clasificando los resultados en tres intervalos, determinados a partir de la amplitud del intervalo, así:

$$
a=\frac{R}{M}
$$

Donde:

$a=$ amplitud

$\mathrm{R}=$ Rango $\rightarrow R=$ Dato mayor - Dato menor

$\mathrm{M}=$ número de intervalos

$a=\frac{1}{3}=0,33$

El análisis factorial confirmatorio, utilizando modelos de ecuaciones estructurales (SEM), obtuvo con el índice de bondad de ajuste un ajuste de 0.658 ; con la raíz cuadrada de la media de los residuos al cuadrado (RMR), un ajuste bueno, pero no perfecto con 0.11 ; y con el error de aproximación cuadrático medio (RMSEA) bajo con 0.112. Los resultados recogidos en la encuestas fueron analizados en Microsoft Excel (Microsoft Corp; E.E.U.U.) y en el paquete estadístico IBM SPSS Statistics, versión 20 (IBM Corp., Armonk, E.E.U.U.) y su interface "Amos Graphics".

\section{Resultados}

Luego de la aplicación de la encuesta, se obtuvo un índice de vulnerabilidad del municipio de Pasto para el año 2012 de 0,334, ubicándolo en el nivel medio de vulnerabilidad. A través de la desagregación del índice, se observó que la dimensión de previsión y percepción es la que tuvo el índice más alto $(0,762)$, lo cual significa que los hogares, en general, no tienen capacidad de reacción frente a situaciones adversas y que los hogares no planean y no preparan estrategias de superación en caso de emergencia, y que en la mayoría de los casos esperan la protección del Estado, aun cuando su percepción sobre éste no indicó aspectos positivos. La dimensión económica tuvo un índice de vulnerabilidad de $(0,569)$, lo que indica que en el municipio de Pasto, las políticas no apuntaron a la generación de empleo que proporcionara mayor estabilidad a los trabajadores. La dimensión ambiental y geográfica obtuvo un valor de $(0,264)$, lo que significa que no hubo proyectos de concientización hacia el cuidado y preservación de los recursos naturales, así como de adecuadas estrategias orientadas a la reubicación de los hogares, localizados en zonas de riesgos de desastres naturales. La dimensión social tuvo un índice de $(0,186)$, es decir, que las políticas públicas no actuaron adecuadamente en los campos de la educación, la salud, la vivienda y el espacio público. Por último, la dimensión demográfica fue de $(0,135)$, es decir, que hubo aspectos particulares que modificaron la estructura del hogar, relacionados con factores internos propios de cada hogar como la jefatura femenina, la dependencia demográfica, la monoparentalidad, entre otros (donde las políticas no pueden intervenir de forma directa) y, a causa de factores externos propios del sistema y las condiciones del país, tales como: la violencia, el secuestro, el desplazamiento, entre otros (Figura 3).

El 58\% de los hogares se ubicaron dentro del nivel bajo de vulnerabilidad, mientras que el $42 \%$ restante se ubicó en el nivel medio, mientras el municipio de Pasto alcanzó un nivel medio de vulnerabilidad. A medida que se avanza en el estrato socioeconómico, los niveles de vulnerabilidad medio van disminuyendo. Los hogares del estrato 1 y 2 obtuvieron el mayor nivel de vulnerabilidad, con 0,367 y 0,352 respectivamente. Por su parte, los hogares del estrato 4 y 5 tuvieron apenas un nivel de vulnerabilidad con una diferencia de 0,011, lo cual se debió a que las características o condiciones de vida de estos hogares fueron similares, mientras los del estrato 2 obtuvieron un nivel superior al de los hogares de la zona rural. Por consiguiente, este resultado indica que la vulnerabilidad, no se pudo ligar a la pobreza, pues hubo factores que ubicaron como vulnerable a un hogar no pobre. Ahora bien, se observó que los hogares de la zona rural tuvieron un nivel de vulnerabilidad menor que el de los hogares de los estratos 
Figura 3

Índice de vulnerabilidad por dimensiones.

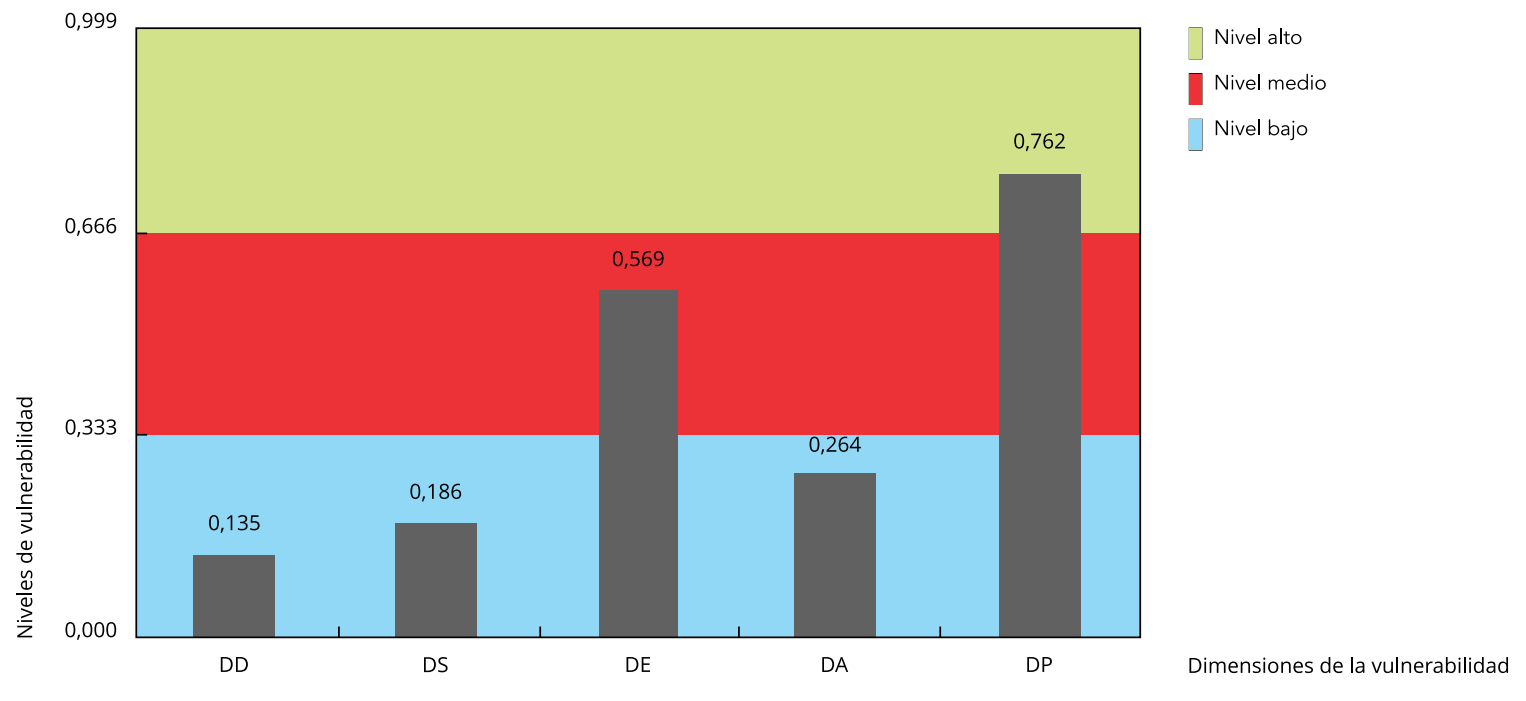

DA: dimensión ambiental-geográfica; DD: dimensión demográfica; DE: dimensión económica; DP: dimensión de previsión y percepción;

DS: dimensión social.

1 y 2 de la zona urbana, lo cual evidencia que a pesar de compartir condiciones de vida similares, los hogares de la zona rural se encuentran en un estado más favorable, debido en gran medida a que el costo de vida es menor (Figura 4).

La dimensión de previsión y percepción presentó mayor vulnerabilidad en la zona rural, y en el estrato 1 de la zona urbana, evidenciando que los hogares del municipio no tienden a formular planes de prevención y mitigación de sucesos que atenten contra su bienestar. Esta situación obedece a que los hogares con condiciones económicas más precarias tienen menor capacidad para afrontar situaciones difíciles o negativas. Dentro de esta dimensión, la variable más significativa resulto ser seguros, lo cual indicó que en los hogares más pobres, por costos económicos o falta de cultura, no contaron con seguros que les permita sobreponerse a sucesos negativos. Asimismo, las variables políticas, y afiliación a pensión, indican que no hubo confianza en el accionar del Estado, lo cual no contribuye al impacto de políticas públicas dirigidas a estas poblaciones por la falta de interés y colaboración con el gobierno. En cuanto a la variable pasado, que hizo referencia a la percepción sobre la vida de la generación pasada en el hogar, se observó que tiene un nivel de vulnerabilidad alto, lo que implica que en términos de percepción la generación que antecede al jefe de hogar presentaron condiciones de vida mejor.

La dimensión económica conservó una relación inversa con el estrato socioeconómico en la zona urbana, pasando de 0,657 en el estrato 1, a 0,336 en el estrato 5. Esta situación evidentemente correspondió a la diferencia entre las condiciones económicas de un hogar de estrato bajo y uno alto. Curiosamente, en los hogares de la zona rural, la dimensión económica presento un menor nivel de vulnerabilidad, en comparación con los hogares de los estratos 1 y 2; esta situación demostró que los hogares rurales se encontraban en mejores condiciones económicas. Dentro de esta dimensión la variable más representativa fue ingresos adicionales, seguida de la variable estabilidad en los ingresos, lo cual explica porque en estratos socioeconómicos bajos, las personas trabajan de manera independiente o dentro de la informalidad. Mientras la variable DE fue la que menor grado de vulnerabilidad presentó, probablemente, porque se redujo en los últimos años el número de miembros por hogar pasando de 3,9 en 2003 a 3,5 en 2012 a nivel nacional. 

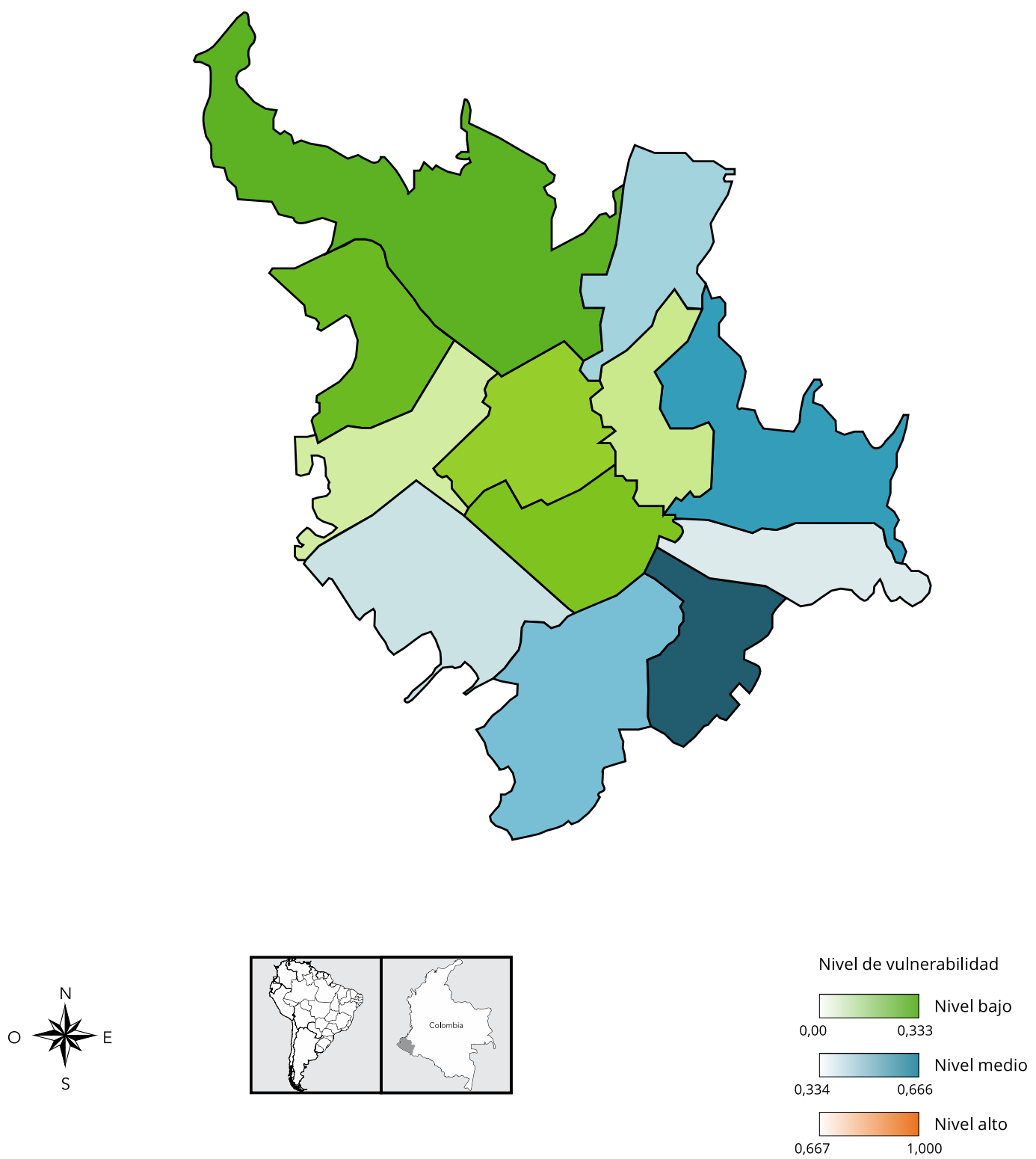

La dimensión ambiental presentó mayor vulnerabilidad en los hogares del estrato 1. Aun así, la vulnerabilidad en esta dimensión mantuvo un comportamiento estable en los demás estratos y en la zona rural. Respecto a la variable, el reciclaje, seguida de contaminación, con un nivel medio de vulnerabilidad fueron las que mayor diferencia significativa presentaron.

Ahora bien, la dimensión social obtuvo el nivel de vulnerabilidad más alto en los hogares de la zona rural y en los hogares urbanos del estrato 2 y 1, esto explica por qué estos hogares acumulan menor capital humano, físico y social, consecuencia de un menor nivel de educación y salud, precarias viviendas y carencia de relaciones sociales. Dentro de esta dimensión, las variables más significativas fueron espacios públicos, tenencia de vivienda (conformando el capital físico), educación y manejo de 
Internet (conformando el capital humano y capital social). Por último, la dimensión demográfica presentó un comportamiento inusual, ya que según los resultados no se pudo definir algún tipo de relación con el estrato socioeconómico.

Las comunas ubicadas en el centro, occidente, noroccidente y nororiente del municipio de Pasto, reflejaron los niveles más bajos de vulnerabilidad. Mientras que las comunas del oriente, sur, suroriente y suroccidente reflejaron niveles medios. Se observó que a medida que las comunas se alejan del centro del municipio, el índice de vulnerabilidad va aumentando, por lo cual algunos de las comunas ubicadas en los extremos tuvieron muchas probabilidades de llegar a un nivel medio de vulnerabilidad.

\section{Discusión}

Cabe mencionar que el índice de vulnerabilidad favorece la evaluación requerida en el debate de las políticas de salud pública 20. El instrumento utilizado en este estudio fue una evaluación individual, que se realizó a través de los proxy means test con limitantes como su costosa aplicación y su incapacidad para identificar un suceso coyuntural o estructural. Sin embargo, tiene ventajas que justifican su cálculo, pues reduce la subjetividad, limita el suministro de información falsa y otorga uniformidad a la evaluación, ya que el propósito de este estudio planteó la vulnerabilidad, pensando en los hogares y para los hogares, pues ellos son el núcleo fundamental de la sociedad. De manera que se concibieron teóricamente en la construcción del indicador tres acciones políticas fundamentales: la prevención, la resistencia y la superación, pues la situación de vulnerabilidad de un hogar puede ser evaluada antes, durante o después de la ocurrencia de un evento negativo. Esto significa que un hogar puede ser vulnerable o puede ya estar vulnerado.

Ahora bien, dado que el escalamiento óptimo asigna cuantificaciones numéricas de acuerdo a los datos proporcionados, cada vez que se repita el procedimiento con otra población los resultados van a cambiar y por ende cambiara el número de variables, y las variables explicativas con que se calcula el índice de vulnerabilidad, tras el análisis factorial, por esta razón no se puede establecer comparaciones con otros grupos poblacionales. Igualmente, el coeficiente de alfa de Cronbach de 0,764 permitió afirmar que el instrumento hizo mediciones estables y consistentes. Mientras que el estadístico KMO y el test de esfericidad de Bartlett aseguraron que fue válido utilizar el análisis factorial. Por medio de las pruebas de bondad de ajuste del modelo, planteado a través del análisis factorial confirmatorio, utilizando SEM, se definió que el modelo propuesto fue medianamente aceptable mediante el nivel de ajuste. De modo que mediante la metodología propuesta fue posible evaluar de manera desagregada el índice de vulnerabilidad de cada dimensión, así como también fue posible determinar cuál de las variables, que componen a cada una de ellas, se encuentra en los niveles más altos de vulnerabilidad, lo cual proporciona un análisis más detallado que va de lo general a lo particular y viceversa.

La recogida y análisis de información demostró que el instrumento es capaz de orientar los planes de desarrollo que permiten focalizar el gasto en las zonas más afectadas del municipio, de modo que permitiría generar estrategias, normas y planes que aporten un mayor número de oportunidades a la población. La metodología planteada puede ser aplicada a nivel país, sin embargo, al aplicarla en una zona específica se genera la no variabilidad en los resultados de algunas variables como desastres naturales. No obstante, dicha variable no se podría suprimir de la metodología, pues en una eventual aplicación, éste es un aspecto que ejerce influencia en la determinación de la vulnerabilidad de los hogares del país.

Se encontró que los hogares del municipio presentan un índice de vulnerabilidad 0,334, ubicándolos en un nivel medio de vulnerabilidad, la cual conserva una relación inversa con el estrato socioeconómico, siendo la dimensión de previsión y percepción y la dimensión económica, las de mayor significancia, estando la primera en un nivel alto de vulnerabilidad y la segunda en un nivel medio (Figura 5). La relación inversa entre el estrato y la vulnerabilidad reafirma el hecho de que la vulnerabilidad está ligada a la pobreza, razón por la cual muchas investigaciones a nivel mundial toman esta última como una variable dependiente, especialmente en la zona urbana donde habita la sociedad del riesgo, ya que hay incapacidad para la cohesión de estructuras sociales, dado el proceso de individualización demoliberal, que se empezó a experimentar con la expansión demográfica de Latinoamérica desde mitad del siglo XX, y su encuadre con la precarización del trabajo, la privatización de los servicios y un estado 


\section{Figura 5}

Nivel de vulnerabilidad por dimensiones según estrato socioeconomico y zona.

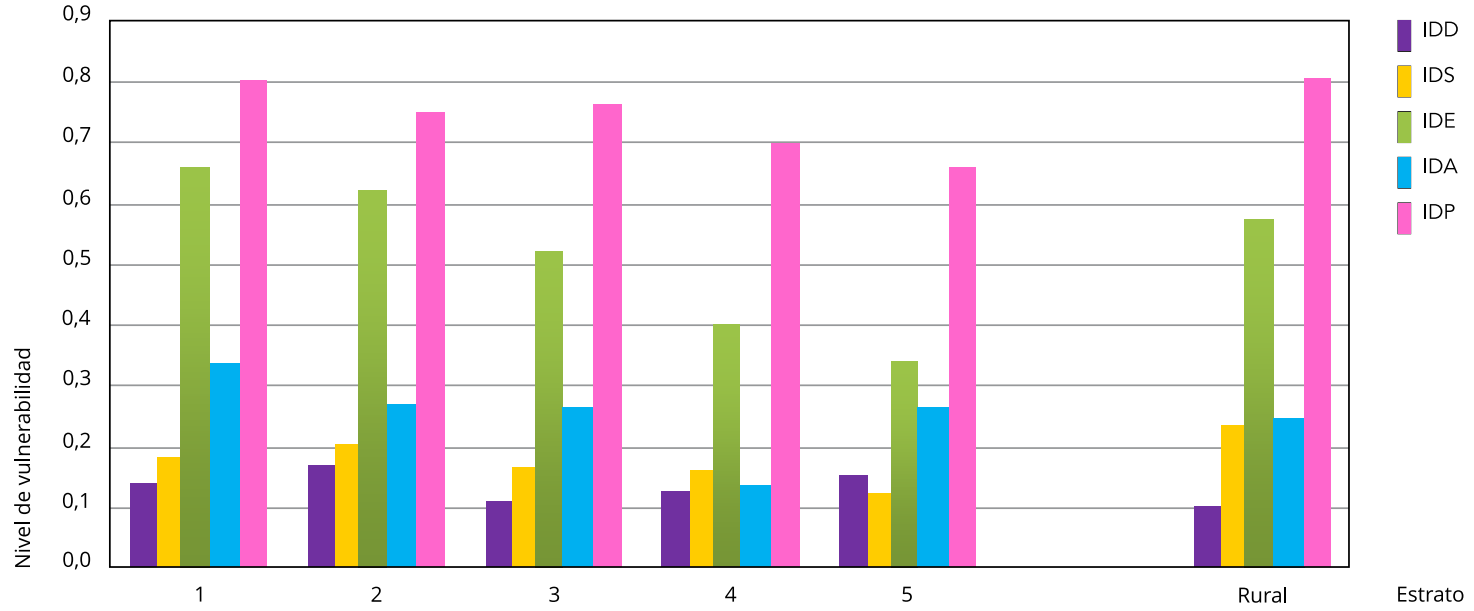

IDA: índice de la dimensión ambiental-geográfica; IDD: índice de la dimensión demográfica; IDE: índice de la dimensión económica; IDP: índice de la dimensión de previsión y percepción; IDS: índice de la dimensión social.

asistencialista 21. Sin embargo, actualmente la variable pobreza se ha ido descartando de la comprensión de la vulnerabilidad, cuando se involucran en las investigaciones los impactos humanos como el cambio climático, y la capacidad de los hogares o comunidades para adaptarse como una herramienta política para la asignación de los recursos del gobierno y el soporte para el desarrollo de actividades económicas alternativas 7 y la diversificación de la agricultura en las zonas rurales ${ }^{4}$. Estos estudios con indicadores ecológicos plantean, a diferencia de este estudio, que la vulnerabilidad ambiental no es el producto de la exposición física al cambio climático u otros peligros, sino que es el producto también de los contextos políticos, económicos y sociales de los hogares 5,22,23; por tanto este estudio es limitado respecto a la comprensión de la vulnerabilidad ambiental, porque la reduce a lo físico. No obstante, explora la vulnerabilidad en los contextos políticos y sociales, sin necesidad de construir índices holísticos que integren variables de capital de los medios de vida, de la percepción del cambio climático, y de los métodos de adaptación. Este estudio está enfocado a las prioridades que contribuyen a la mejora de la equidad y la justicia social de los hogares de una región en particular.

Por otro lado, los hallazgos de este estudio, permiten afianzar la idea de que es necesario un modelo económico sustentable, tal como se ha propuesto en otros estudios en ciudades latinoamericanas 24 , donde incluso en clases sociales medias se ha visto afectada la educación y el mercado laboral como en Argentina y los activos físicos en México 25, o en asuntos tan puntuales como la dieta en la infancia, como se ha demostrado en Brasil 26. El municipio de Pasto es un pequeño centro urbano que como otros 1 que podrían considerarse de menor vulnerabilidad 27 , porque no posee una profunda heterogeneidad entre sus residentes, como sí sucede en grandes urbes como Bogotá o Cali 28, aunque con un riesgo ambiental 29 por la presencia de un volcán activo 30 . Este estudio propone que se explore en Colombia variables como migraciones por motivo de salud, percepción sobre disponibilidad y calidad de los espacios públicos (centros de salud y hospitales), tipo de afiliación a salud, prevención en salud, control de crecimiento y desarrollo en menores de 5 años, percepción sobre problemas futuros como la salud, adquisición de seguros de salud entre los residentes urbanos y rurales y la vulnerabilidad en niveles socioeconómicos bajos, medios y altos, ya que las condiciones materiales de existencia no son suficientes para explicar la vulnerabilidad de los hogares. Lo anterior en vista de que un indicador de vulnerabilidad social en hogares plantea una dinámica socioterritorial que se refleja en el espacio geográfico, donde se fusionan materialidades y acciones, condiciones locales y globales, redes que integran y redes que 
disuelven las acciones de la sociedad; las cuales adquieren contenido si se reconocen los vínculos entre el individuo y la sociedad 31 .

De modo que este estudio demuestra que la pobreza no es la única causante de la vulnerabilidad de los hogares 4,22, y que, por tanto, un hogar no pobre puede presentar algún nivel de vulnerabilidad en aspectos diferentes a los económicos, de ahí que sea válido la creación de un índice de vulnerabilidad multidimensional, como el expuesto en esta investigación, en el que se tuvo en cuenta los aspectos económicos sin restarle importancia a otras dimensiones 13 . Por último, es importante mencionar que los resultados obtenidos a través del índice de vulnerabilidad en cada una de las dimensiones, es concordante con la evidencia empírica de estudios sociales 32,33 que afirman que cuanto más pobre es un hogar, es más vulnerable, dado sus menores niveles de educación, menores ingresos y falta de herramientas para la previsión, pero también es coherente con la evidencia en Colombia que demuestra que alrededor del 18\% de los colombianos son vulnerables a la pobreza sin ser pobres, mientras el $20 \%$ de los hogares colombianos son pobres y tienen una alta probabilidad de permanecer en ella; en conclusión, la vulnerabilidad es más común que la pobreza 25,34 .

\section{Colaboradores}

V. L. Estupiñan-Ferrín, G. F. Guerrero-Díaz y A. Hidalgo-Troya participaron en la concepción y diseño de este trabajo; así como en la recogida, análisis y interpretación de los datos para el mismo y em la redacción del artículo. A. Rocha-Buelvas maquetación y revisión crítica del trabajo, así como del contenido intelectual importante.

\section{Agradecimientos}

Muy especialmente a los profesores Laura Golovaneski, Rubén Kaztman y Gustavo Wilches Chaux por permitir el intercambio de ideas, opiniones y sugerencias. Asimismo, al grupo de investigación Coyuntura Económica y Social (CES) de la Universidad de Nariño y a su ex-director Robert Wilson Ortiz por el apoyo y el respaldo brindado. Convocatoria de investigación "Alberto Quijano Guerrero" de la Vicerrectoría de Investigaciones, Postgrados y Relaciones. 


\section{Referencias}

1. Barata RB, Ribeiro MCS, Cassanti AC; Grupo do Projeto Vulnerabilidade Social no Centro de São Paulo. Social vulnerability and health status: a household survey in the central area of a Brazilian metropolis. Cad Saúde Pública 2011; 27 Suppl 2 :S164-75.

2. Busso G. Vulnerabilidad social: nociones e implicancias de políticas para Latinoamérica a inicios del siglo XXI. In: Seminario Internacional: Las Diferentes Expresiones de la Vulnerabilidad Social en América Latina y el Caribe. Santiago: Comisión Económica para América Latina y el Caribe; 2001. p. 3-34.

3. Golovanesky L. Vulnerabilidad y transmisión intergeneracional de la pobreza. Un abordaje cuantitativo para Argentina en el siglo XXI. Buenos Aires: Universidad de Buenos Aires; 2007.

4. Dumenu WK, Obeng EA. Climate change and rural communities in Ghana: social vulnerability, impacts, adaptations and policy implications. Environ Sci Policy 2016; 55:208-17.

5. Pandey R, Bardsley DK. Social-ecological vulnerability to climate change in the Nepali Himalaya. Appl Geogr 2015; 64:74-86.

6. Abid M, Schilling J, Scheffran J, Zul F. Science of the total environment climate change vulnerability, adaptation and risk perceptions at farm level in Punjab, Pakistan. Sci Total Environ 2016; 547:447-60.

7. Morzaria-Luna HN, Turk-Boyer P, MorenoBaez M. Social indicators of vulnerability for fishing communities in the Northern Gulf of California, Mexico: implications for climate change. Mar Policy 2014; 45:182-93.

8. Sugden F, Maskey N, Clement F, Ramesh V, Philip A, Rai A. Agrarian stress and climate change in the Eastern Gangetic Plains: gendered vulnerability in a stratified social formation. Glob Environ Change 2014; 29:258-69.

9. Ebi KL, Bowen K. Extreme events as sources of health vulnerability: drought as an example. Weather and Climate Extremes 2016; 11:95-102.
10. Powell BJ, Proctor EK, Glisson C, Kohl PL, Raghavan R, Brownson RC, et al. A mixed methods multiple case study of implementation as usual in children's social service organizations: study protocol. Implement Sci 2013; 8:92.

11. Comisión Económica para América Latina y el Caribe. Marco conceptual sobre activos, vulnerabilidad y estructura de oportunidades. Montevideo: Comisión Económica para América Latina y el Caribe; 1999.

12. Programa de las Naciones Unidas para el Desarrollo. La reducción de riesgos de desastres: un desafío para el desarrollo. New York: Programa de las Naciones Unidas para el Desarrollo; 2004.

13. Ayuso Álvarez I, Cadena Vargas E. Índice de vulnerabilidad social en los países de la OCDE. Madrid: Universidad Autónoma de Madrid; 2006. (Economic Analysis Working Paper Series. Working Paper, 1/2006).

14. Nuñez J, Espinosa S. No siempre pobres, no siempre ricos: vulnerabilidad en Colombia. Bogotá: Universidad de los Andes; 2005. (Documento CEDE 2005-15).

15. Dirección de Desarrollo Social, Departamento Nacional de Planeación. Mecanismos de focalización: cuatro estudios de caso. Bogotá: Dirección de Desarrollo Social, Departamento Nacional de Planeación; 2007.

16. Comisión Económica para América Latina y el Caribe; Oficina para el Cono Sur de América Latina y el Caribe, Organización Internacional del Trabajo. Coyuntura laboral en América Latina y el Caribe: los programas de transferencias condicionadas y el mercado laboral. Santiago: Comisión Económica para América Latina y el Caribe; 2014. (Boletín CEPAL/OIT).

17. Red de Observatorios Regionales del Mercado de Trabajo. Diagnóstico socioeconómico y del mercado de trabajo en la ciudad de Pasto. Pasto: Red de Observatorios Regionales del Mercado de Trabajo; 2012. 
18. Departamento Administrativo Nacional de Estadísticas. Boletín: censo general 2005. Bogotá: Departamento Administrativo Nacional de Estadísticas; 2010.

19. Con M, Susini S, Catalá S, Quinteros S. Índice de vulnerabilidad social (IVS): documento metodológico. Buenos Aires: Dirección de Investigación y Estadística, Ministerio de Educación; 2009.

20. Buchalla CM, Paiva V. Da compreensão da vulnerabilidade social ao enfoque multidisciplinar. Rev Saúde Pública 2002; 36:117-9.

21. Imilan W. Repensar la producción contemporánea del hábitat, vulnerabilidad y pobreza en la ciudad. Revista INVI 2010; 25(Part 1):9-14.

22. Beroya-Eitner MA. Ecological vulnerability indicators. Ecol Indic 2016; 60:329-34.

23. Liu H-L, Willems P, Bao A-M, Wang L, Chen X. Effect of climate change on the vulnerability of a socio-ecological system in an arid area. Glob Planet Change 2016; 137:1-9.

24. Ezcurra E, Mazari M, Pisanty I, Aguilar AG. La ciudad de México: entre la vulnerabilidad ambiental y la sustentabilidad. Economía, Sociedad y Territorio 2008; 8:1067-78.

25. Galassi GL, González LM. Vulnerability factors in the middle class: evidence for Argentina and Mexico after the crisis of the 1990s. Frontera Norte 2003; 24:89-116.

26. Bortolini GA, Vitolo MR, Gubert MB, Santos LMP. Iniquidades sociais influenciam a qualidade e a diversidade da dieta de crianças brasileiras de 6 a 36 meses. Cad Saúde Pública 2015; 31:2413-24.

27. Programa Naciones Unidas para el Desarrollo. Pasto: ciudad región. Bases para una política de inclusión productiva para la población en situación de pobreza y vulnerabilidad. Bogotá: Programa Naciones Unidas para el Desarrollo; 2010. 34 p.
28. Lampis A. ¿Qué ha pasado con la vulnerabilidad social en Colombia? Conectar libertades instrumentales y fundamentales. Sociedad y Economia 2010; (19):229-61.

29. Jerez Heredia EK. Amenaza volcánica en Colombia: antecedentes y perspectivas. Bucaramanga: Universidad Industrial de Santander; 2009.

30. Narváez O, Viteri MA. Plan departamental de gestión del riesgo nariño 2008-2018. Pereira: Universidad Tecnológica de Pereira; 2009.

31. Santos M. Por una geografía nueva. Madrid: Espasa Universidad; 1990.

32. Castillo Vargas A, Castro-Chaves X. El rostro de la violencia social y estructural: la delincuencia y la pobreza como expresiones distintas de una vulnerabilidad común. Revista de Ciencias Sociales 2011; III-IV:113-24.

33. Knight L, Hosegood V, Timæus IM. The South African disability grant: influence on HIV treatment outcomes and household well-being in. Dev South Af 2013; 30:135-47.

34. Núñez J, Espinosa S. Determinantes de la pobreza y la vulnerabilidad. Bogotá: Departamento Nacional de Planeación; 2005. 


\section{Abstract}

This study's objective was to measure the vulnerability index of families in the municipality of Pasto, Colombia. In a sample of 270 families of all socioeconomic strata (239 urban and 31 rural), a confidential survey was conducted using the vulnerability index, consisting of five dimensions: demographic, social, economic, environmental, and geography, forecasting, and prevention. The families in strata 1 and 2 and the houses in the center, west, northwest, and northeast of the urban area showed high vulnerability, even higher than those in rural areas. Fifty percent of the families showed low vulnerability and $42 \%$ medium vulnerability. The poorest families were the most vulnerable, but poor families were also vulnerable in the municipality of Pasto.

Poverty; Vulnerable Populations;

Social Conditions

\section{Resumo}

O objetivo do estudo foi medir a taxa de vulnerabilidade das famílias no Município de Pasto, Colômbia. Em uma amostra de 270 famílias de todos os estratos socioeconômicos, 239 em áreas urbanas e 31 nas zonas rurais, uma pesquisa confidencial foi realizada usando-se o indice de vulnerabilidade e consiste em cinco dimensões: demográfica, social, econômica, ambiental e geográfica, e previsão e prevenção. As famílias dos estratos $1 \mathrm{e} 2$, bem como as casas do centro, oeste, noroeste e nordeste da área urbana do município apresentaram alta vulnerabilidade, ainda maior do que aqueles das áreas rurais. Cinquenta por cento das famílias estavam localizadas em baixa vulnerabilidade e $42 \%$ eram de nível médio. As famílias mais pobres são as mais vulneráveis, no entanto, as famílias pobres também eram vulneráveis no Município de Pasto.

Pobreza; Populações Vulneráveis;

Condições Sociais

Recibido el 27/Jul/2015

Versión final presentada el 29/Mar/2016

Aprobado el 28/Abr/2016 